\title{
Coronal plane alignment in orthotic treatment of adolescent idiopathic scoliosis: evaluation of balance parameters in orthotic outcomes
}

\author{
Nicole Yeh, James H Wynne*, Timothy Hresko \\ From 7th International Conference on Conservative Management of Spinal Deformities \\ Montreal, Canada. 20-22 May 2010
}

\begin{abstract}
Introduction
There are different ways to quantify coronal plane alignment on a radiograph, and there is a need to compare the relationship of these different methods with orthotic outcome. The purpose of this study is to investigate the relationship between methods of measuring spinal alignment and orthotic outcomes for the Boston Brace method of treating adolescent idiopathic scoliosis.
\end{abstract}

\section{Materials and methods}

This is a retrospective study examining coronal plane spine radiographs of 27 adolescents diagnosed with idiopathic scoliosis and prescribed a Boston Brace-style thoracolumbosacral orthosis. Cobb angle, decompensation of C7, and Overall Balance Summation (OBS) (Carlson and Smith, 2007) were measured on pre-brace, in-brace, and immediately post-brace $\mathrm{x}$-rays for each subject. Curve progression was defined as a greater than 5 degree increase in Cobb angle or greater than $10 \mathrm{~mm}$ increase in OBS. A statistical analysis was performed to determine significant differences between bracing success and failure groups for in pre-brace and in-brace curve values.

\section{Results}

The results show that curves whose balance worsened had significantly lower decompensation and OBS values versus curves whose balance did not progress during bracing. There was no significant difference in pre-brace decompensation or OBS for curves whose Cobb angle progressed compared to those who did not progress. There was no significant difference in in-brace balance values for either outcome group. However, when balance in-brace was better than initial out-of-brace balance, the curve tended to maintain the improved balance throughout bracing period. There was no difference in the relationship to outcomes when balance was measured by decompensation of C7 or OBS.

\section{Discussion}

This study shows that either decompensation of $\mathrm{C} 7$ or Overall Balance Summation may be used to quantify coronal plane spinal balance. There was no distinction in the relationship of these two parameters to bracing outcomes. This study suggests that bracing may control coronal plane balance, but a long term study with a control group is needed. No conclusions can be made about the relationship between Cobb angle and decompensation or OBS.

\section{Conclusion}

Overall Balance Summation may be used to quantify coronal plane balance of adolescent idiopathic scoliosis. However, its relationship to curve progression and the amount of desirable in-brace correction is still not understood.

Published: 10 September 2010 